\title{
Improvement of diagnostic efficiency in distinguishing the benign and malignant thyroid nodules via conventional ultrasound combined with ultrasound contrast and elastography
}

\author{
MEI-JUAN LIU ${ }^{1 *}$, YAN-MING MEN ${ }^{1 *}$, YONG-LIN ZHANG ${ }^{2}$, YU-XI ZHANG $^{1}$ and HAO LIU ${ }^{3}$ \\ ${ }^{1}$ Department of Ultrasound, The Affiliated Yantai Yuhuangding Hospital of Qingdao University; \\ ${ }^{2}$ Department of Ultrasound, Yantai Affiliated Hospital of Binzhou Medical University; ${ }^{3}$ Department of Ultrasound, \\ Yantai Hospital of Traditional Chinese Medicine, Yantai, Shandong 264001, P.R. China
}

Received January 25, 2017; Accepted April 24, 2017

DOI: $10.3892 / 01.2017 .6245$

\begin{abstract}
We aimed to evaluate the diagnostic values of conventional ultrasound (US), ultrasound contrast (UC) and ultrasound elastography (UE) in distinguishing the benign and malignant thyroid nodules. A total of 100 patients with thyroid nodules receiving operative treatment were selected; they underwent the conventional US, UE and UC examinations before operation, respectively. The nodules received pathological examination after operation to distinguish benign from malignant lesions. The sensitivity, specificity and diagnostic accordance rate of each diagnostic method was evaluated by receiver operating characteristic (ROC) curve, and the area under the curve (AUC) of ROC was calculated. The manifestations of malignant thyroid nodules in conventional US examination were mostly the hypoecho, heterogeneous echo, irregular shape, unclear boundary, aspect ratio $<1$, microcalcification and irregular peripheral echo halo, and there were statistically significant differences compared with the benign nodules $(\mathrm{P}<0.05)$. UE showed that the differences between benign and malignant nodules in 2, 3 and 4 points were statistically significant $(\mathrm{P}<0.05)$. The manifestations of malignant nodules in UC were mostly the irregular shape, obscure boundary, no obvious enhancement, heterogeneous enhancement and visible perfusion defects, and there were statistically significant differences compared with the benign nodules $(\mathrm{P}<0.05)$. ROC curve showed that both sensitivity and specificity of UE and UC were superior to those of conventional US. AUC was the largest (AUC $=0.908)$ and the diagnostic value
\end{abstract}

Correspondence to: Dr Hao Liu, Department of Ultrasound, Yantai Hospital of Traditional Chinese Medicine, 39 Xingfu Road, Zhifu, Yantai, Shandong 264001, P.R. China

E-mail: dr74sa@163.com

${ }^{*}$ Contributed equally

Key words: conventional ultrasound, ultrasound contrast, ultrasonic elastography, thyroid nodules, diagnostic value was the highest in the conventional US combined with UE and UC. Conventional US combined with elastography and UC can significantly improve the sensitivity, specificity and accuracy of diagnosis of benign and malignant thyroid nodules.

\section{Introduction}

Conventional ultrasound (US) examination has become one of the preferred imaging examination methods of thyroid disease, but thyroid diseases are complex and diverse; in the two-dimensional ultrasonogram, there are overlapping benign and malignant nodules, and some nodules have the features of both benign nodular and malignant nodules, known as an uncertain nodule and it is difficult to determine its nature only from the ultrasonogram, so there are still some limitations on its differential diagnosis, directly affecting the choices of clinical treatment and surgical methods, which requires the physicians to make a preliminary qualitative diagnosis of lesions, so as to provide the reference basis for clinicians (1-4).

Ultrasound elastography (UE) is one of the new ultrasonic examination techniques, and its theoretical basis is that the soft and hard degree of lesions is displayed on the screen through the information of elastic surface, and the examiner determines the soft and hard degree of lesions by observing the color information on the screen, and then speculates whether the lesions are benign or malignant (5-8). In recent years, ultrasound contrast (UC) has become one of the most rapidly-developed examinations, and its principle is that the accumulation of contrast agent in the lesion tissues is observed to determine the sensitivity of lesion tissues to the contrast agent, thus improving the sensitivity and specificity of diagnosis (9-12). In the actual clinical application, UC is still in the exploratory stage in the diagnosis of thyroid tumor lesions, and there are also relative limitations of elastography diagnosis, namely a certain crossing and overlapping phenomena of elastic coefficients between different tissue structures.

This study aimed to compare the accuracy of different methods through evaluating the combined application of ultrasonic examination techniques, so as to provide a more effective diagnostic basis for distinguishing benign from malignant thyroid nodules and provide the basis for clinical work. 


\section{Patients and methods}

Subjects. One hundred patients with thyroid nodules receiving an ultrasonic examination and operation in the Affiliated Yantai Yuhuangding Hospital of Qingdao University from January 2015 to October 2016 were selected, including 33 males and 67 females aged 25-75 years with an average age of $40.26 \pm 8.03$ years. Patients received the conventional US, UE and UC examinations before operation. After operation, patients were divided into a benign group and a malignant group according to pathological results. There were 125 nodules (70 benign, 55 malignant) in conventional US and UE, and 122 nodules ( 65 benign, 57 malignant) in UC. This study was approved by the Ethics Committee of the Affiliated Yantai Yuhuangding Hospital. Signed written informed consents were obtained from all participants before the study.

Instruments and reagents. HI VISION Preirus color Doppler US diagnostic instrument (Hitachi Medical Systems, Tokyo, Japan; probe frequency, 9-12 MHz; mechanical index, 0.08) was used; the probe frequency of UE was $5-12 \mathrm{MHz}$, with real-time tissue elastography technique and built-in real-time tissue elastography analysis software. SonoVue (Bracco SpA, Milan, Italy) was used as the contrast agent.

Examination methods. The lesion was diagnosed by two physicians with years of experience in US diagnosis. After the thyroid nodules were found via conventional US, the site, number, size, border, shape and internal echo of the nodules were observed and recorded. The distribution of the blood vessels inside the nodules was observed. Then under the elastic mode, elastography examination was conducted for thyroid for the elastic classification of nodules. Finally, under the UC mode, $1.6 \mathrm{ml}$ suspension of contrast agent was injected into the superficial vein of left elbow, followed by $5 \mathrm{ml}$ normal saline. The tissue perfusion was observed in time. The dynamic contrast images were recorded for 2-4 min, and the time-intensity curve was analyzed in one region of interest within the thyroid nodule, and the peak time, peak intensity, curve apex and area under the curve (AUC) of nodules were recorded using the automatic tracking quantitative analysis software.

Diagnostic criteria. UC image analysis included the enhancement degree and enhancement uniformity. According to the color distribution and proportion in the lesion, the hardness of lesion in the US real-time elastography was divided into 5 grades. Grade 0: red, green and blue in the lesion area; Grade I: uniform green in the lesion area; Grade II: the green area in the lesion area $>50 \%$; Grade III: the blue area in the lesion area $=50-90 \%$; Grade IV: blue in almost all the lesion area and the blue area $>90 \%$. Grade 0 , I and II were determined as the benign thyroid nodule, while Grade III and IV were determined as the malignant benign nodule.

Statistical analysis. All statistics were performed using SPSS 19.0 software (IBM, Armonk, NY, USA). Chi-square test was performed for the comparison between groups. The receiver operating characteristic (ROC) curve was drawn, and AUC of ROC was calculated. $\mathrm{P}<0.05$ suggested that the difference was statistically significant.

\section{Results}

Conventional US examination. There were statistically significant differences in the comparisons of nodular echo, nodal echo, shape, boundary definition, aspect ratio, calcification degree and regularity of peripheral echo halo between malignant nodules and benign nodules $(\mathrm{P}>0.05$; Table I), but there was no statistically significant difference in the posterior attenuation between the two groups $(\mathrm{P}>0.05$; Table I).

UE examination. The UE score of malignant thyroid nodules were usually 3 points, and that of benign nodules were usually 2 points; the differences were statistically significant between benign nodules and malignant nodules in 2, 3 and 4 points $(\mathrm{P}<0.05$; Table II), but there was no statistically significant difference between them in 1 point $(\mathrm{P}>0.05$; Table II).

UC examination. Malignant thyroid nodules were usually characterized by the irregular shape, obscure boundary, no obvious enhancement, heterogeneous enhancement and visible perfusion defects, while benign nodules were usually characterized by the regular shape, clear boundary, enhanced perfusion, homogeneous enhancement and no perfusion defects. There were statistically significant differences between them $(\mathrm{P}<0.05$; Table III).

Comparisons of quantitative parameters of the benign and malignant thyroid nodules. The quantitative parameter curve tip of malignant nodules was significantly higher than that of benign nodules, and the difference was statistically significant $(\mathrm{P}<0.05$; Table IV), but there were no statistically significant differences in the initial time of enhancement, time to peak, peak intensity and AUC between benign and malignant nodules ( $\mathrm{P}>0.05$; Table IV).

Comparisons of accuracy of different US examination parameters. ROC curve showed that AUC was the largest (AUC $=0.759$; Table V) when 3-5 diagnostic indexes were satisfied in conventional US, and the accuracy rate was up to 77.4\%; AUC was the largest $(\mathrm{AUC}=0.648$; Table V) when the score $\geq 3$ points in elastography, and the accuracy rate was up to $68.5 \%$; and $\mathrm{AUC}$ was the largest $(\mathrm{AUC}=0.746$; Table $\mathrm{V})$ when 1-2 diagnostic indexes were satisfied in UC, and the accuracy rate was up to $75.2 \%$. The sensitivity and specificity of UE and UC were better, and AUC was the largest $(\mathrm{AUC}=0.908$; Table V) in the combined application of both US examinations, and the sensitivity and specificity of diagnosis were 96.6 and $94.2 \%$, respectively, and the accuracy rate was up to $92.6 \%$ (Table V).

\section{Discussion}

Thyroid cancer is one of the common malignant tumors, showing an increasing trend in recent years, and its incidence ranks the seventh in cancer in China currently (13). According to the data collected by the National Cancer Registry, the incidence of thyroid cancer in females in the past 10 years has increased by $18 \%$ on average with the largest rise in all cancers $(14,15)$. Therefore, how to improve the accuracy rate of diagnosis of thyroid nodules and avoid the excessive treatment 
Table I. Conventional ultrasound features of benign and malignant thyroid nodules (n, \%).

\begin{tabular}{|c|c|c|c|c|}
\hline Characteristic & Benign nodule & Malignant nodule & $\chi^{2}$ & P-value \\
\hline Nodule echo & & & 7.342 & 0.036 \\
\hline Non-hypoecho & $14(20.00)$ & $4(7.27)$ & & \\
\hline Hypoecho & $56(80.00)$ & $51(92.73)$ & & \\
\hline Nodule echo condition & & & 10.407 & 0.004 \\
\hline Homogeneous & $16(22.86)$ & $3(5.45)$ & & \\
\hline Heterogeneous & $54(77.14)$ & $52(94.55)$ & & \\
\hline Shape & & & 14.156 & 0.003 \\
\hline Regular & $57(81.43)$ & $30(54.55)$ & & \\
\hline Irregular & $13(18.57)$ & $25(45.45)$ & & \\
\hline Boundary & & & 28.532 & $<0.001$ \\
\hline Clear & $62(88.57)$ & $23(41.82)$ & & \\
\hline Unclear & $8(11.43)$ & $32(58.18)$ & & \\
\hline Aspect ratio & & & 6.814 & 0.048 \\
\hline$<1$ & $64(91.43)$ & $43(78.18)$ & & \\
\hline$\geq 1$ & $6(8.57)$ & $12(21.82)$ & & \\
\hline Calcification & & & 16.169 & $<0.001$ \\
\hline No or huge & $56(80.00)$ & $23(41.82)$ & & \\
\hline Micro & $14(20.00)$ & $32(58.18)$ & & \\
\hline Peripheral echo halo & & & 19.923 & $<0.001$ \\
\hline Regular & $60(85.71)$ & $26(47.27)$ & & \\
\hline Irregular & $10(14.29)$ & $29(52.73)$ & & \\
\hline Rear attenuation & & & 9.418 & 0.509 \\
\hline Yes & $63(90.00)$ & $47(85.45)$ & & \\
\hline No & $7(10.00)$ & $8(14.55)$ & & \\
\hline
\end{tabular}

Table II. Elasticity scores of benign and malignant thyroid nodules (n).

\begin{tabular}{|c|c|c|c|c|}
\hline \multirow[b]{2}{*}{ Score } & \multicolumn{2}{|c|}{ Group } & \multirow[b]{2}{*}{$\chi^{2}$} & \multirow[b]{2}{*}{ P-value } \\
\hline & Benign nodule & Malignant nodule & & \\
\hline 1 & 3 & 1 & 1.347 & 1.608 \\
\hline 2 & 56 & 20 & 23.126 & 0.004 \\
\hline 3 & 9 & 30 & 14.259 & $<0.001$ \\
\hline 4 & 2 & 4 & 6.358 & 0.043 \\
\hline
\end{tabular}

is a research hotspot for scholars in China and other countries currently. The characteristics of conventional ultrasonography, UC and UE of thyroid nodules were analyzed, so as to evaluate their diagnostic values in the benign and malignant thyroid nodules.

Literature reports and this study indicate that the main manifestations of malignant nodules included the solid, hypoecho, heterogeneous echo, obscure boundary, unsmooth boundary, microcalcification and internal blood flow $(14,15)$. However, in the examination, the sensitivity and specificity of two-dimensional ultrasonic diagnosis of thyroid nodule are often not very satisfactory due to the diverse manifestations of thyroid nodules and lack of experience of US physicians, so the conventional US has certain misdiagnosis in clinical practice.

UC can detect the blood supply in the tumor more sensitively and enhance the nodules with poor blood supply after contrast, which can provide certain information for clinical identification (9-12). The results of this study showed that malignant thyroid nodules were mostly manifested with irregular shape, obscure boundary, no significant enhancement, heterogeneous enhancement and visible perfusion defects, while benign nodules were mostly manifested with regular shape, clear boundary, enhanced perfusion, homogeneous enhancement and no perfusion defects. UC quantitative analysis technique can help draw the time-intensity curve of 
Table III. Ultrasound contrast image features (n, \%).

\begin{tabular}{|c|c|c|c|c|}
\hline Characteristic & Benign nodule & Malignant nodule & $\chi^{2}$ & P-value \\
\hline Boundary & & & 28.646 & $<0.001$ \\
\hline Clear & $49(75.38)$ & $16(28.07)$ & & \\
\hline Unclear & $16(24.62)$ & $41(71.93)$ & & \\
\hline Shape & & & 32.175 & $<0.001$ \\
\hline Regular & $50(76.92)$ & $14(24.56)$ & & \\
\hline Irregular & $15(23.08)$ & $43(75.44)$ & & \\
\hline Perfusion intensity & & & 31.205 & $<0.001$ \\
\hline Enhanced & $48(73.85)$ & $22(38.60)$ & & \\
\hline Non-enhanced & $17(26.15)$ & $35(61.40)$ & & \\
\hline Homogeneous enhancement & & & 16.768 & 0.004 \\
\hline Yes & $42(64.62)$ & $23(40.35)$ & & \\
\hline No & $23(35.38)$ & $34(59.65)$ & & \\
\hline Perfusion defects & & & 20.719 & $<0.001$ \\
\hline Yes & $14(21.54)$ & $38(66.67)$ & & \\
\hline No & $51(78.46)$ & $19(33.33)$ & & \\
\hline
\end{tabular}

Table IV. Comparisons of quantitative parameters of benign and malignant thyroid nodules (mean \pm standard deviation).

\begin{tabular}{|c|c|c|c|c|}
\hline Characteristic & Benign nodule & Malignant nodule & $\chi^{2}$ & P-value \\
\hline Start time (sec) & $14.05 \pm 3.97$ & $14.97 \pm 4.56$ & 1.406 & 0.365 \\
\hline Peak time (sec) & $39.03 \pm 5.49$ & $36.14 \pm 5.98$ & 0.743 & 0.109 \\
\hline Peak intensity $(\mathrm{dB})$ & $43.99 \pm 5.07$ & $46.08 \pm 5.84$ & 0.862 & 0.314 \\
\hline Curve angle (l/sec) & $0.14 \pm 0.03$ & $0.28 \pm 0.07$ & 3.541 & 0.042 \\
\hline Area under curve $(1 / \mathrm{sec})$ & $4.75 \pm 2.49$ & $4.92 \pm 2.83$ & 0.905 & 0.327 \\
\hline
\end{tabular}

Table V. Indexes of different ultrasound examinations in the diagnosis of benign and malignant thyroid nodules (\%).

\begin{tabular}{|c|c|c|c|c|c|c|}
\hline Characteristic & Sensitivity & Specificity & Positive predictive value & Negative predictive value & Accuracy & AUC \\
\hline \multicolumn{7}{|l|}{ Conventional US } \\
\hline Match 1-2 & 96.8 & 16.3 & 44.6 & 83.0 & 51.6 & 0.584 \\
\hline Match 3-5 & 64.1 & 87.6 & 81.4 & 77.7 & 77.4 & 0.759 \\
\hline Match >5 & 4.2 & 97.7 & 82.7 & 63.8 & 63.8 & 0.577 \\
\hline \multicolumn{7}{|l|}{ UE scores } \\
\hline$\geq 2$ & 99.4 & 3.9 & 45.5 & 100.0 & 43.2 & 0.515 \\
\hline$\geq 3$ & 52.1 & 80.8 & 70.3 & 66.9 & 68.5 & 0.648 \\
\hline$\geq 4$ & 7.2 & 95.7 & 68.2 & 58.1 & 59.2 & 0.503 \\
\hline \multicolumn{7}{|l|}{$\mathrm{UC}$} \\
\hline Match 1-2 & 65.3 & 84.8 & 78.1 & 72.6 & 75.2 & 0.746 \\
\hline Match $\geq 3$ & 59.5 & 88.6 & 78.4 & 72.5 & 74.6 & 0.727 \\
\hline \multicolumn{7}{|l|}{ Methods } \\
\hline Conventional US & 45.8 & 74.4 & 55.9 & 67.4 & 62.8 & 0.581 \\
\hline UE & 86.5 & 89.6 & 84.8 & 91.4 & 86.9 & 0.834 \\
\hline $\mathrm{UC}$ & 89.8 & 92.7 & 87.6 & 93.0 & 91.3 & 0.885 \\
\hline $\mathrm{UE}$ and UC & 96.6 & 94.2 & 87.7 & 96.2 & 92.6 & 0.908 \\
\hline
\end{tabular}

AUC, area under curve; US, ultrasound; UE, ultrasound elastography; UC, ultrasound contrast. 
contrast agent with the change of time during the UC, and obtain the relevant quantitative parameters. This study found that there were no statistically significant differences in the initial time of enhancement, time to peak, peak intensity and AUC between benign and malignant nodules; the curve apex of malignant nodules was significantly higher than that of benign nodules, which was basically consistent with the results of Friedrich-Rust et al (16). There have been no unified diagnostic criteria of the UC in distinguishing benign and malignant thyroid nodules; due to different factors, UC technique still has some limitations, so the UE should be combined to confirm the nature of lesion.

UE can obtain the tissue elasticity information that cannot be obtained by conventional imaging mode, which is a new extension of conventional color Doppler ultrasonography (5). UE has been widely used in clinical diagnosis of liver, prostate, mammary gland and thyroid with the elastic characteristics of organism as the theoretical basis (6-8). In general, the hardness of malignant thyroid nodules is higher than that of benign thyroid nodules, and the hardness of lesion is positively correlated with its oncogenous rate. The results of this study showed that the UE score of malignant thyroid nodules was mostly 3 points, and that of benign nodules was mostly 2 points; and the differences were statistically significant between benign and malignant nodules in 2, 3 and 4 points, which was consistent with the results of studies in other countries.

ROC curve suggested that the sensitivity, specificity and accuracy of conventional US combined with UC and UE were higher than those of the single application of conventional US in the diagnosis of benign and malignant thyroid nodules, indicating that the conventional US combined with UC and UE can further improve the sensitivity, specificity and accuracy of examination. With the continuous development of US examination techniques and the continuous improvement of examination methods, we believe that the combined US examination techniques will continue to be used clinically in the near future, offering help for the choice of clinical operation methods and postoperative follow-up treatment.

In conclusion, conventional US combined with UC and UE can be used as effective means to distinguish benign from malignant thyroid nodules. Conventional US combined with UC and elastography improves the accuracy of distinguishing benign from malignant thyroid nodules, providing a more effective diagnostic basis for distinguishing benign from malignant thyroid nodules and the basis for clinical selection of surgical methods, which has a broad application prospect.

\section{References}

1. Giannetti A, Randisi P, Stumpo M and Coratti F: Diagnosis of one small bowel tumor: the role of conventional ultrasound and elastography. J Ultrasound 19: 57-60, 2014.

2. Bertolotto M, Serafini G, Sconfienza LM, Lacelli F, Cavallaro M, Coslovich A, Tognetto D and Cova MA: The use of CEUS in the diagnosis of retinal/choroidal detachment and associated intraocular masses - preliminary investigation in patients with equivocal findings at conventional ultrasound. Ultraschall Med 35: 173-180, 2014.
3. Zhao RN, Zhang B, Yang X, Jiang YX, Lai XJ and Zhang XY: Logistic regression analysis of contrast-enhanced ultrasound and conventional ultrasound characteristics of sub-centimeter thyroid nodules. Ultrasound Med Biol 41: 3102-3108, 2015.

4. Song KD, Lee MW, Rhim H, Cha DI, Chong Y and Lim HK: Fusion imaging-guided radiofrequency ablation for hepatocellular carcinomas not visible on conventional ultrasound. AJR Am J Roentgenol 201: 1141-1147, 2013.

5. Nowicki A and Dobruch-Sobczak K: Introduction to ultrasound elastography. J Ultrason 16: 113-124, 2016.

6. Samir AE: The role and value of ultrasound elastography in the evaluation of thyroid nodules. Cancer 124: 765-766, 2016.

7. Korrungruang $P$ and Boonsarngsuk V: Diagnostic value of endobronchial ultrasound elastography for the differentiation of benign and malignant intrathoracic lymph nodes. Respirology: Jan 19, 2017 (Epub ahead of print)

8. Reginelli A, Urraro F, di Grezia G, Napolitano G, Maggialetti N, Cappabianca S, Brunese L and Squillaci E: Conventional ultrasound integrated with elastosonography and B-flow imaging in the diagnosis of thyroid nodular lesions. Int J Surg 12 (Suppl 1): 117-122, 2014.

9. Fan Z, Li Y, Yan K, Wu W, Yin S, Yang W, Xing B, Li X and Zhang X: Application of contrast-enhanced ultrasound in the diagnosis of solid pancreatic lesions - a comparison of conventional ultrasound and contrast-enhanced CT. Eur J Radiol 82: 1385-1390, 2013.

10. Wang Y, Liao J, Qi W, Xie L and Li Y: Predictive value of conventional ultrasound and contrast-enhanced ultrasound in early recurrence of hepatocellular carcinoma after surgical resection. Ultrasound Med Biol 42: 1042-1048, 2016.

11. Yan CX, Luo ZY, Liu XM, Huang PT, Mo GQ, Hong YR, Wen Q, Pan MQ and Weng HF: Ultrasonic scores of conventional ultrasound and ultrasound elastography in the diagnosis of thyroid nodular lesions. Zhonghua Yi Xue Za Zhi 93: 1630-1633, 2013 (In Chinese).

12. Li F and Luo H: Comparative study of thyroid puncture biopsy guided by contrast-enhanced ultrasonography and conventional ultrasound. Exp Ther Med 5: 1381-1384, 2013.

13. Liu Y, Lin Z, Sheng C, Zhu Y, Huang Y, Zhong N, Jia Z and Qu S: The prevalence of thyroid nodules in northwest China and its correlation with metabolic parameters and uric acid. Oncotarget: Jan 18, 2017 (Epub ahead of print).

14. Choi YJ, Baek JH, Park HS, Shim WH, Kim TY, Shong Y and Lee JH: A computer-aided diagnosis system using artificial intelligence for the diagnosis and characterization of thyroid nodules on ultrasound: initial clinical assessment. Thyroid: Jan 10, 2017 (Epub ahead of print).

15. Paschou SA, Vryonidou A and Goulis DG: Thyroid nodules: a guide to assessment, treatment and follow-up. Maturitas 96: 1-9, 2017.

16. Friedrich-Rust M, Sperber A, Holzer K, Diener J, Grünwald F, Badenhoop K, Weber S, Kriener S, Herrmann E, Bechstein WO, et al: Real-time elastography and contrast-enhanced ultrasound for the assessment of thyroid nodules. Exp Clin Endocrinol Diabetes 118: 602-609, 2010. 